\title{
Development of efficient GPU parallelization of WRF Yonsei University planetary boundary layer scheme
}

\author{
M. Huang ${ }^{1}$, J. Mielikainen ${ }^{1}$, B. Huang ${ }^{1}$, H. Chen ${ }^{1}$, H.-L. A. Huang ${ }^{1}$, and M. D. Goldberg ${ }^{2}$ \\ ${ }^{1}$ Space Science and Engineering Center, University of Wisconsin-Madison, Madison, WI 53706, USA \\ ${ }^{2}$ NOAA/JPSS, Lanham, MD 20706, USA \\ Correspondence to: B. Huang (bormin@ssec.wisc.edu)
}

Received: 17 September 2014 - Published in Geosci. Model Dev. Discuss.: 21 November 2014

Revised: 11 June 2015 - Accepted: 3 September 2015 - Published: 30 September 2015

\begin{abstract}
The planetary boundary layer (PBL) is the lowest part of the atmosphere and where its character is directly affected by its contact with the underlying planetary surface. The PBL is responsible for vertical sub-grid-scale fluxes due to eddy transport in the whole atmospheric column. It determines the flux profiles within the well-mixed boundary layer and the more stable layer above. It thus provides an evolutionary model of atmospheric temperature, moisture (including clouds), and horizontal momentum in the entire atmospheric column. For such purposes, several PBL models have been proposed and employed in the weather research and forecasting (WRF) model of which the Yonsei University (YSU) scheme is one. To expedite weather research and prediction, we have put tremendous effort into developing an accelerated implementation of the entire WRF model using graphics processing unit (GPU) massive parallel computing architecture whilst maintaining its accuracy as compared to its central processing unit (CPU)-based implementation. This paper presents our efficient GPU-based design on a WRF YSU PBL scheme. Using one NVIDIA Tesla K40 GPU, the GPU-based YSU PBL scheme achieves a speedup of $193 \times$ with respect to its CPU counterpart running on one CPU core, whereas the speedup for one CPU socket (4 cores) with respect to $1 \mathrm{CPU}$ core is only $3.5 \times$. We can even boost the speedup to $360 \times$ with respect to $1 \mathrm{CPU}$ core as two K40 GPUs are applied.
\end{abstract}

\section{Introduction}

The science of meteorology explains observable weather events, and its application is weather forecasting. Nowadays, sophisticated instruments are used for observations from upper air atmosphere. The collected quantitative data about the current state of the atmosphere are then used to predict future states. This requires the aid of equations of fluid dynamics and thermodynamics that are based on laws of physics, chemistry, and fluid motion. The weather research and forecasting (WRF) (http://www.wrf-model.org/ index.php) model meets such a requirement, which is a simulation program consisting of several different physical processes and dynamic solvers. It is designed to serve the needs of both operational forecasting and atmospheric research.

The WRF system supports some of the best possible models for weather forecasting. Nevertheless, a continual challenge for timely weather forecasting is forecast model execution speed. This is a challenge even with the fastest supercomputers, in particular for severe weather events.

With the advent of graphics processing unit (GPU) architectures, the execution speed of weather forecasting models can be greatly increased by taking advantage of the parallelism feature of GPUs. GPUs have hundreds of parallel processor cores for execution on tens of thousands of parallel threads. Furthermore, GPUs possess several merits, such as low cost, low power, large bandwidth, and high performance. These make GPUs more effective than a massively parallel system built from commodity central processing units (CPUs). Usage of GPUs has been applied very successfully to deal with numerous computational problems in various domains, for instance, porting marine ecosystem model spin-up 


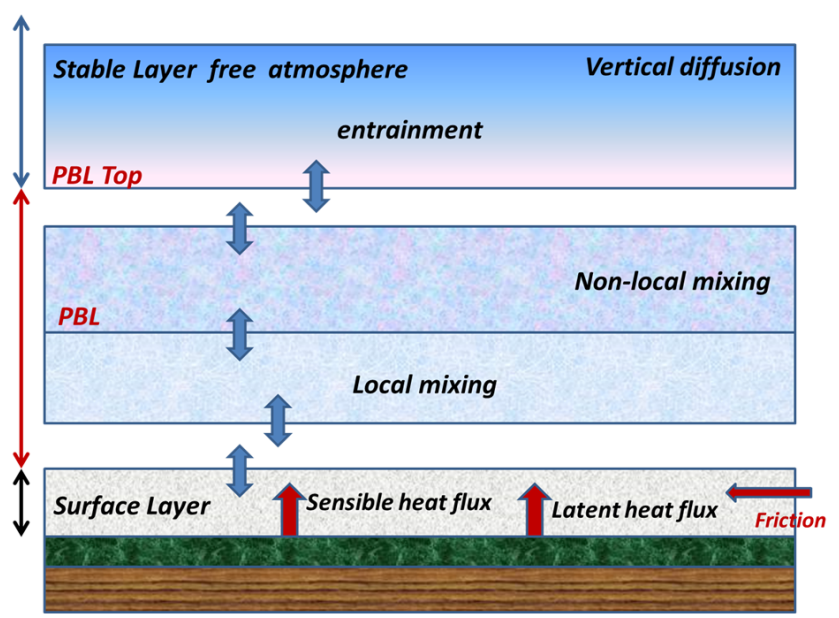

Figure 1. Illustration of PBL process (Overview of WRF Physics, 2014).

using transport matrices to GPUs (Siewertsen et al., 2013), GPU-accelerated long-wave radiation scheme of the rapid radiative transfer model for general circulation (RRTMG) models (Price et al., 2014), advances in multi-GPU smoothed particle hydrodynamics simulations (Rustico et al., 2014), speeding up the computation of WRF double moment 6class microphysics scheme with GPU (Mielikainen et al., 2013), real-time implementation of the pixel purity index algorithm for end-member identification on GPUs (Wu et al., 2014), fat vs. thin threading approach on GPUs: application to stochastic simulation of chemical reactions (Klingbeil et al., 2012), ASAMgpu V1.0 - a moist fully compressible atmospheric model using GPUs (Horn, 2012), GPU acceleration of predictive partitioned vector quantization for ultra-spectral sounder data compression (Wei, 2011), clusters vs. GPUs for parallel automatic target detection in remotely sensed hyperspectral images (Paz et al., 2010), and a GPUaccelerated wavelet decompression system with set partitioning in hierarchical trees (SPIHT) and Reed-Solomon decoding for satellite images (Song et al., 2011), to name several.

To expedite weather analysis research and forecasting, we have put tremendous efforts into developing an accelerated implementation of the entire WRF model using a GPU massive parallel architecture whilst maintaining its accuracy as compared to its CPU-based implementation. This study develops an efficient GPU-based design on the Yonsei University (YSU) planetary boundary layer (PBL) scheme, which is one of the physical models in WRF. The PBL is responsible for vertical sub-grid-scale fluxes due to eddy transports in the whole atmospheric column. It determines the flux profiles within the well-mixed boundary layer and the more stable layer above, and thus provides an evolutionary model of atmospheric temperature, moisture (including clouds), and horizontal momentum in the entire atmospheric column.
This paper is structured as follows. Section 2 describes YSU PBL scheme. Section 3 outlines the GPU hardware specification as well as a brief description of the basis of the Compute Unified Device Architecture (CUDA) computing engine for GPUs. The GPU-based implementation is also given in Sect. 3. The development of optimizing the GPUbased YSU PBL scheme is presented in Sect. 4. Summary and future work is given in Sect. 5 .

\section{YSU PBL scheme}

The scheme of YSU is one of the PBL models in WRF. The PBL process is illustrated in Fig. 1. Based on Hong et al. (2006), a brief description of the YSU PBL scheme is presented below.

\subsection{Mixed-layer diffusion}

The momentum diffusivity coefficient is formulated based on the work done in Troen et al. (1986), Hong et al. (1996), and Noh et al. (2003):

$K_{m}=k w_{s} z\left(1-\frac{z}{h}\right)^{\mathrm{p}}$,

where $\mathrm{p}$ is the profile shape exponent taken to be $2, k$ the von Karman constant (at 0.4), $z$ the height from the surface, and $h$ the PBL height. The mixed-layer velocity scale is given as

$w_{s}=\left(u_{*}^{3}+\varphi_{m} k w_{* b}^{3} z / h\right)^{1 / 3}$,

where $u_{*}$ is the surface frictional velocity scale, $\varphi_{m}$ the wind profile function evaluated at the top of the surface layer, and $w_{* b}$ the convective velocity scale for the moist air which is defined as

$w_{* b}=\left[\left(g / \theta_{v a}\right)\left(w^{\prime} \theta_{v}^{\prime}\right)_{0} h\right]^{1 / 3}$.

The counter-gradient term for $\theta$ and momentum is given by

$\lambda_{c}=b \frac{\left(\overline{w^{\prime} \theta_{v}^{\prime}}\right)_{0}}{w_{s 0} h}$,

where $\left(\overline{w^{\prime} \theta_{v}^{\prime}}\right)_{0}$ is the corresponding surface flux for $\theta, u$, and $v$, and $b$ the coefficient of proportionality which will be derived below. The mixed-layer velocity scale $w_{s 0}$ is defined as the velocity at $z=0.5 h$ in Eq. (2).

The eddy diffusivity for temperature and moisture $K_{t}$ is computed from $K_{m}$ in Eq. (1) by using the relationship of the Prandtl number (Noh et al., 2003), which is given by

$\operatorname{Pr}=1+\left(\operatorname{Pr}_{0}-1\right) \exp \left[-3(z-\varepsilon h)^{2} / h^{2}\right]$,

where $P r_{0}=\left(\varphi_{t} / \varphi_{m}+b k \varepsilon\right)$ is the Prandtl number at the top of the surface layer given by Troen et al. (1986) and Hong et al. (1996). The ratio of the surface layer height to the PBL height, $\varepsilon$, is specified to be 0.1 . 
To satisfy the compatibility between the surface layer top and the bottom of the PBL, identical profile functions are used to those in surface layer physics. First, for unstable and neutral conditions $\left.\left(\overline{\left(w^{\prime} \theta_{v}^{\prime}\right.}\right)_{0} \geq 0\right)$,

$\varphi_{m}=\left(1-16 \frac{0.1 \mathrm{~h}}{L}\right)^{-1 / 4}$ for $u$ and $v$,

$\varphi_{t}=\left(1-16 \frac{0.1 h}{L}\right)^{-1 / 2}$ for $\theta$ and $q$,

while for the stable regime $\left(\left(\overline{w^{\prime} \theta_{v}^{\prime}}\right)_{0}<0\right)$,

$\varphi_{m}=\varphi_{t}=\left[1+5 \frac{0.1 h}{L}\right]$

where $h$ is, again, the PBL height, and $L$ the MoninObukhov length scale. To determine the factor $b$ in Eq. (3), the exponent of $-1 / 3$ is chosen to ensure the free convection limit. Typically $L$ ranges from -50 to 0 in unstable situations. Therefore, we can use the following approximation:

$\varphi_{m}=\left(1-16 \frac{0.1 h}{L}\right)^{-1 / 4} \simeq\left(1-8 \frac{0.1 h}{L}\right)^{-1 / 3}$

Following the work in Noh et al. (2003) and Moeng et al. (1994), the heat flux amount at the inversion layer is expressed by

$\left(\overline{w^{\prime} \theta^{\prime}}\right)_{h}=-e_{1} w_{m}^{3} / h$,

where $e_{1}$ is the dimensional coefficient $\left(=4.5 \mathrm{~m}^{-1} \mathrm{~s}^{2} \mathrm{~K}\right)$, $w_{m}$ the velocity scale based on the surface layer turbulence $\left(w_{m}^{3}=w_{*}^{3}+5 u_{*}^{3}\right)$, and the mixed-layer velocity scale for the dry air $w_{*}=\left[\left(g / \theta_{a}\right)\left(\overline{w^{\prime} \theta_{0}^{\prime}}\right) h\right]^{1 / 3}$. Using a typical value of $\theta_{a}$ at $300 \mathrm{~K}$, the gravity at $10 \mathrm{~ms}^{-2}$, and the limit of $u_{*}=0$ in the free convection limit, Eq. (8) can be generalized for the moist air with a non-dimensional constant, which can be expressed by

$\left(\overline{w^{\prime} \theta_{v}^{\prime}}\right)_{h}=-0.15\left(\frac{\theta_{v a}}{g}\right) w_{m}^{3} / h$,

where $w_{m}$ considers the water vapor driven virtual effect for buoyancy flux. Given the buoyancy flux at the inversion layer, Eq. (9), the flux at the inversion layer for scalars $\theta$ and $q$, and vector quantities $u$ and $v$, are proportional to the change in each variable at the inversion layer:

$\left(\overline{w^{\prime} \theta^{\prime}}\right)_{h}=\left.w_{\mathrm{e}} \Delta \theta\right|_{h}$,

$\left(\overline{w^{\prime} q^{\prime}}\right)_{h}=\left.w_{\mathrm{e}} \Delta q\right|_{h}$,

$\left(\overline{w^{\prime} u^{\prime}}\right)_{h}=\left.\operatorname{Pr}_{h} w_{\mathrm{e}} \Delta u\right|_{h}$,

$\left(\overline{w^{\prime} v^{\prime}}\right)_{h}=\left.\operatorname{Pr}_{h} w_{\mathrm{e}} \Delta v\right|_{h}$,

respectively. Here $w_{\mathrm{e}}$ is the entrainment rate at the inversion layer, which is expressed by

$w_{\mathrm{e}}=\frac{\left(\overline{w^{\prime} \theta_{v}^{\prime}}\right)_{h}}{\left.\Delta \theta_{v}\right|_{h}}$,

where the maximum magnitude of $w_{\mathrm{e}}$ is limited to $w_{m}$ to prevent excessively strong entrainment in the presence of too small of a jump in $\theta_{v}$ in the denominator. The Prandtl number at the inversion layer $P r_{h}$ is set as 1. Meanwhile, the flux for the liquid water substance at the inversion layer is assumed to be zero.

Following Hong et al. (1996), $h$ is determined by checking the stability between the lowest model level and levels above considering the temperature perturbation due to surface buoyancy flux, which is expressed by

$\theta_{v}(h)=\theta_{v a}+\theta_{T}\left[=a \frac{\left(\overline{w^{\prime} \theta_{v}^{\prime}}\right)_{0}}{w_{s 0}}\right]$,

where $a$ is set to 6.8 , the same as the $b$ factor in Eq. (3). In Eq. (2), $\theta_{T}$ ranges less than $1 \mathrm{~K}$ under clear-sky condition where $\theta_{v}(h)$ is the virtual potential temperature at $h$. The quantity $a$ is an important parameter in the new scheme. Numerically, $h$ is obtained by two steps. First, $h$ is estimated by

$h=R i b_{\mathrm{cr}} \frac{\theta_{v a}|U(h)|^{2}}{g\left[\theta_{v}(h)-\theta_{s}\right]}$

without considering $\theta_{T}$, where $R i b_{\mathrm{cr}}$ is the critical bulk Richardson number, $U(h)$ the horizontal wind speed at $h, \theta_{v a}$ the virtual potential temperature at the lowest model level, $\theta_{v}(h)$ the virtual potential temperature at $\theta_{v}(h)$, and $\theta_{s}$ the near-surface temperature. This estimated $h$ is utilized to compute the profile functions in Eqs. (5)-(7), and to compute the $w_{s 0}$, which is estimated to be the value at $z=h / 2$ in Eq. (2). Second, using $w_{s 0}$ and $\theta_{T}$ in Eq. (12), $h$ is revised by checking the bulk stability, Eq. (12), between the surface layer (lowest model level) and levels above. With the revised $h$ and $w_{s 0}, K_{m}$ is obtained by Eq. (1), entrainment terms in Eqs. (9)-(11), and $K_{t}$ by the Prandtl number in Eq. (4). The counter gradient correction terms for $\theta$ in Eq. (4) are also obtained by Eq. (3). 


\subsection{Free atmosphere diffusion}

The local diffusion scheme, the so-called local $K$ approach (Louis, 1979), is utilized for free atmospheric diffusion above the mixed layer $(z>h)$. In this scheme, the effects of local instabilities in the environmental profile and the penetration of entrainment flux above $h$ irrespective of local stability are both taken into account within the entrainment zone and the local $K$ approach above.

In Noh (2003), the diffusion coefficients for mass $(t: \theta, q)$ and momentum $(m: u, v)$ are expressed by

$\begin{aligned} K_{t_{-} \text {ent }} & =\frac{-\left(\overline{w^{\prime} \theta_{v}^{\prime}}\right)_{h}}{\left(\partial \theta_{v} / \partial z\right)_{h}} \exp \left[-\frac{(z-h)^{2}}{\delta^{2}}\right], \\ K_{m_{-} \text {ent }} & =\operatorname{Pr}_{h} \frac{-\left(\overline{w^{\prime} \theta_{v}^{\prime}}\right)_{h}}{\left(\partial \theta_{v} / \partial z\right)_{h}} \exp \left[-\frac{(z-h)^{2}}{\delta^{2}}\right],\end{aligned}$

and the thickness of the entrainment zone can be estimated as

$\delta / h=d_{1}+d_{2} R i_{\text {con }}^{-1}$,

where $w_{m}$ is the velocity scale for the entrainment, $R i_{\text {con }}$ the convective Richardson number at the inversion layer: $R i_{\text {con }}=\left[\left(g / \theta_{v a}\right) h \Delta \theta_{v_{-} \text {ent }}\right] / w_{m}^{2}$, and constants $d_{1}$ and $d_{2}$ are set as 0.02 and 0.05 , respectively.

Following Noh (2003) and Louis (1979), the vertical diffusivity coefficients for momentum $(m: u, v)$ and mass $(t ; \theta, q)$ above $h$ are represented as

$K_{m \_l o c, t} \__{-}$loc $=l^{2} f_{m, t}(R i g)\left(\frac{\partial U}{\partial z}\right)$,

in terms of the mixing length $l$, the stability function $f_{m, t}($ Rig $)$, and the vertical wind shear, $|\partial U / \partial z|$. The stability functions $f_{m, t}$ are represented in terms of the local gradient Richardson number Rig. For the non-cloudy layer,

$\operatorname{Rig}=\frac{g}{\theta_{v}}\left[\frac{\partial \theta_{v} / \partial z}{(\partial U / \partial z)^{2}}\right]$.

For the cloudy air, Rig is modified for reduced stability within cloudy air, which is expressed by (Durran et al., 1982)

$\operatorname{Rig}_{c}=\left(1+\frac{L_{v} q_{v}}{R_{d} T}\right)\left[\operatorname{Rig}-\frac{g^{2}}{|\partial U / \partial z|^{2}} \frac{1}{c_{p} T} \frac{(A-B)}{(1+A)}\right]$,

where $A=L_{v}^{2} q_{v} / c_{p} R_{v} T^{2}$ and $B=L_{v} q_{v} / R_{d} T$. The computed Rig is truncated to -100 to prevent unrealistically unstable regimes. The mixing length scale $l$ is given by

$\frac{1}{l}=\frac{1}{k_{z}}+\frac{1}{\lambda_{0}}$,

where $k$ is the von Karman constant $(=0.4), z$ the height from the surface, and $\lambda_{0}$ is the asymptotic length scale $(=150 \mathrm{~m})$ (Kim et al., 1992). The stability function, $f_{m, t}($ Rig $)$, differs for stable and unstable regimes. The stability formulas from the National Centers for Environmental Prediction (NCEP) Markov random field (MRF) model (Betts et al., 1996) are used. For the stably stratified free atmosphere $($ Rig $>0)$,

$f_{m, t}(R i g)=\frac{1}{(1+5 R i g)^{2}}$,

and the Prandtl number is given by

$\operatorname{Pr}=1.0+2.1=$ Rig.

For the neutral and unstably stratified atmosphere $(R i g \leq 0)$,

$f_{t}($ Rig $)=1-\frac{8 \text { Rig }}{1+1.286 \sqrt{- \text { Rig }}}$,
$f_{m}($ Rig $)=1-\frac{8 \text { Rig }}{1+1.746 \sqrt{- \text { Rig }}}$.

For the entrainment zone above $h$, the diffusivity is determined by geometrically averaging the two different diffusivity coefficients from Eqs. (13) and (14), and is expressed by

$K_{m, t}=\left(K_{m, t_{-} \text {ent }} K_{m}, t_{-} \text {loc }\right)^{1 / 2}$.

Equation (21) represents not only the entrainment, but also the free atmospheric mixing when the entrainment above the bottom of the inversion layer is induced by vertical wind shear at PBL top. With the diffusion coefficients and counter-gradient correction terms computed in Eqs. (1)(21), the diffusion equations for all prognostic variables $\left(C, u, v, \theta, q_{v}, q_{c}, q_{i}\right)$ expressed as, for example for $C$,

$\frac{\partial C}{\partial t}=\frac{\partial}{\partial z}\left[k_{c}\left(\frac{\partial C}{\partial z}-\gamma_{c}\right)-\left(\overline{w^{\prime} c^{\prime}}\right)_{h}\left(\frac{z}{h}\right)^{3}\right]$

and can be solved by an implicit numerical method (Bright et al., 2002) that has been built in the WRF model. Here $C$ is heat capacity, $u$ and $v$ are horizontal velocity components, $\theta$ is the potential temperature, $q_{v}, q_{c}$, and $q_{i}$ are the mixing ratios of water vapor, cloud, and cloud ice, respectively. Note that the term $-\left(\overline{w^{\prime} c^{\prime}}\right)_{h}\left(\frac{z}{h}\right)^{3}$ is an asymptotic entrainment flux term at the inversion layer and is not included in the MRF PBL model (Hong et al., 1996).

Since there are no interactions among horizontal grid points, the WRF YSU PBL scheme is highly suited to massively parallel processing and great speed advantage can be expected. What follows is a presentation of our GPU-based development on YSU PBL scheme.

\section{GPU hardware specification}

\subsection{GPU device specification and basis of CUDA engine}

We developed the massively parallel GPU version of the YSU PBL scheme using NVIDIA Tesla K40 GPUs 


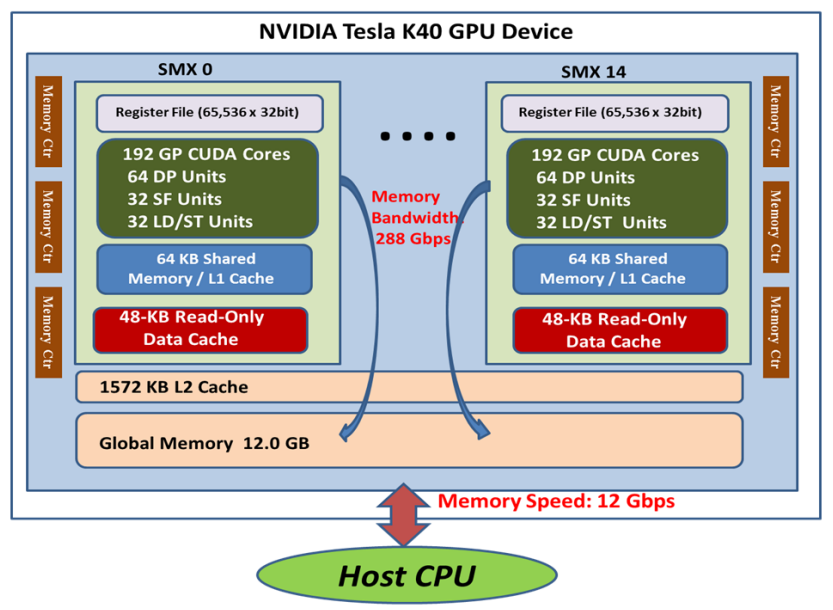

Figure 2. Schematic diagram of hardware specification of one NVIDIA Tesla K40 GPU employed in our study.

(NVIDIA, 2013a, b), while Intel Xeon CPU E5-2603 at $1.8 \mathrm{GHz}$ is used for executing its counterpart CPU-based program for speedup comparison. The clock frequencies of memory and GPU processor (in boost mode) are at 3004 and $875 \mathrm{MHz}$ for K40, respectively. One Tesla K40 GPU has 15 streaming multiprocessors (SMX). Each SMX units has 192 CUDA cores, amounting to 2880 cores. The hardware specification employed in our study is depicted in Fig. 2. In contrast, our CPU system has two sockets, each of which has 4 cores.

CUDA $\mathrm{C}$ is an extension to the $\mathrm{C}$ programming language and which offers a direct programming in the GPUs. It is designed such that its construction allows for execution in a manner of data-level parallelism. A CUDA program is arranged into two parts: a serial program running on the CPU and a parallel part running on the GPU, where the parallel part is called a kernel. The driver, in the C language, distributes a large number of copies of the kernel into available multiprocessors and executes them simultaneously. The CUDA parallel program automatically utilizes more parallelism on GPUs when there are more processor cores available. A CUDA program consists of three computational phases: transmission of data into the global memory of the GPU, execution of the CUDA kernel, and delivery of results from the GPU into the memory of the CPU.

From the viewpoint of CUDA programming, a thread is the basic atomic unit of parallelism. Threads are organized into a three-level hierarchy. The highest level is a grid, which consists of thread blocks. A grid is a three-dimensional (3D) array of thread blocks. A domain of two-dimensional (2D) horizontal grid points, $433 \times 308$, is adopted in the YSU PBL scheme (see Sect. 3.2), which implies that $433 \times 308$ threads are required if one GPU is used. Each thread executes the whole numerical calculation of equations described in Sect. 2. Given the block size (i.e., number of threads per

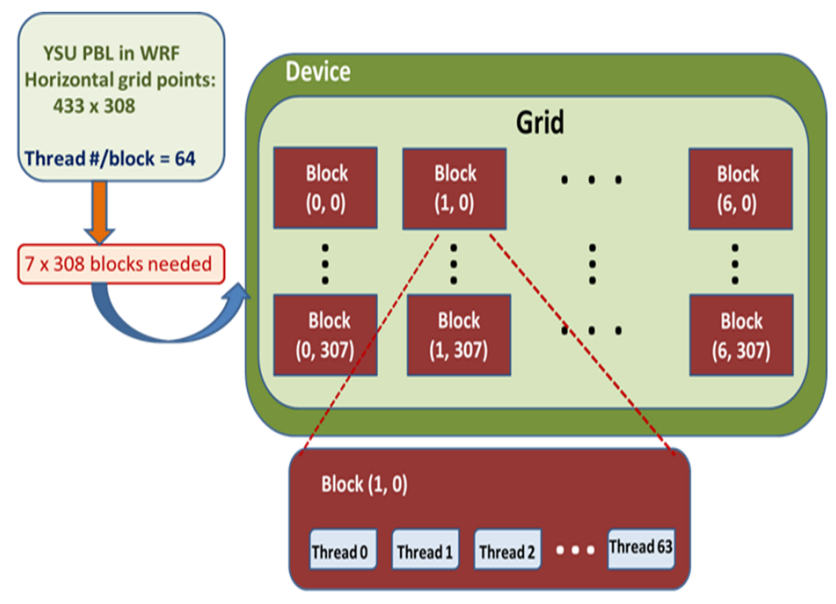

Figure 3. Three-level thread hierarchy of a device for one GPU utilized in our study: threads, thread block, and grids of block.

block) of 64 available, this suggests that one needs $7 \times 308$ blocks. Figure 3 illustrates the three-level thread hierarchy of a device for one GPU that was implemented in our study. Thread blocks implement coarse-grained scalable data parallelism and they are executed independently, which permits them to be scheduled in any order across any number of cores. This allows the CUDA program to scale with the number of processors.

The execution of CUDA programs can be achieved more efficiently in GPUs if the global memory is maximized and the number of data transactions are minimized. This can be accomplished through the global memory access by every 16 threads grouped together and coalesced into one or two memory transactions. This is the so-called coalesced memory access, which can be more effective if adjacent threads load or store contiguous data, and are aligned in memory. To enable the coalesced global memory access, the function in the NVIDIA CUDA library cudaMallocPitch is used to pad the data, which would enhance the data transfer sizes between host and device.

Threads of 32 are arranged together in execution, which are called a warp, while global memory loads and stores by half of a warp (i.e., 16 threads). A CUDA program group inside a multiprocessor issues the same instruction to all the threads in a warp. Different global memory accesses are coalesced by the device in as few as one memory transaction when the starting address of the memory access is aligned and the threads access the data sequentially. An efficient use of the global memory is one of the essences of a high performance CUDA kernel.

\subsection{GPU-based implementation}

The current WRF programs are written in Fortran. To develop a GPU-based and parallel implementation, the Fortran programs of YSU PBL scheme are first translated to standard 


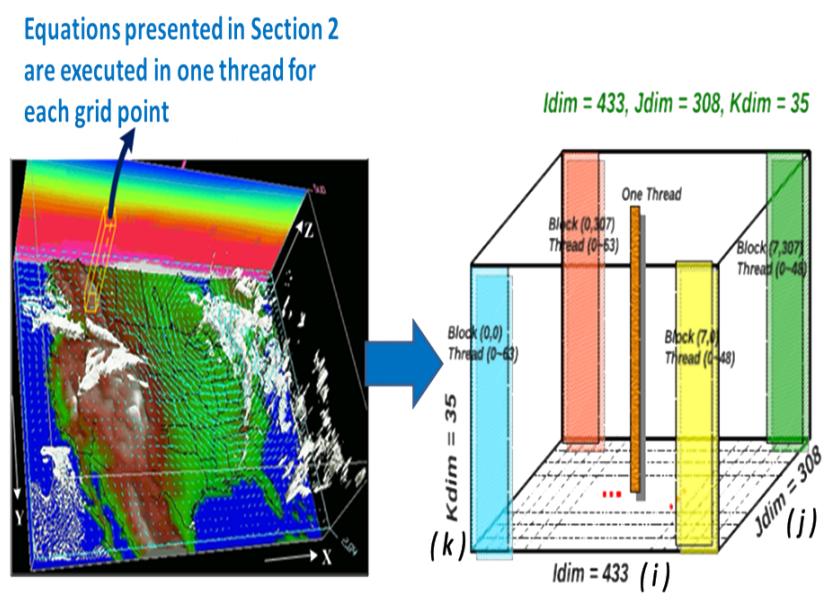

Figure 4. Mapping of the CONUS domain onto one GPU threadblock-grid domain, where the size of the CONUS domain is $433 \times 308$ horizontal grid points with 35 vertical levels.

C programs, followed by converting the $\mathrm{C}$ into CUDA $\mathrm{C}$ that can run on GPUs efficiently. Three major reasons for doing this in this way are (i) to ensure correct results, (ii) to make the difference between $\mathrm{C}$ and CUDA C implementations to be very small, and (iii) to allow conversion from $\mathrm{C}$ programs to CUDA C programs in a short time.

To test whether the programming of the YSU PBL scheme is correct, we used a CONtinental United States (CONUS) benchmark data set, a $12 \mathrm{~km}$ resolution domain, collected on 24 October 2001 (CONUS, 2008). This is a $48 \mathrm{~h}$ forecast over the continental USA capturing the development of a strong baroclinic cyclone and a frontal boundary that extends from north to south across the entire USA. The WRF domain is a geographic region of interest discretized into a 2-D grid points parallel to the ground, which are labeled as $(i, j)$ in the WRF codes and in the following discussion. Each grid point deals with multiple levels, corresponding to various vertical heights in the atmosphere, which is denoted as $k$ in both the programs and in the discussion presented below. The size of the CONUS $12 \mathrm{~km}$ domain is $433 \times 308$ horizontal grid points with 35 vertical levels. Figure 4 exemplifies the mapping of CONUS domains onto one GPU thread-block-grid domain.

In generating correct $\mathrm{C}$ programs from Fortran, followed by the conversion from $\mathrm{C}$ to CUDA $\mathrm{C}$, considerable care must be taken. First of all, Fortran array indexing uses nonzero indices as the first index and these have to be converted into C/CUDA $\mathrm{C}$ arrays using zero-based indexing. That is, the first index value of the Fortran arrays in WRF codes is 1 or non-zero value, while that of a C/CUDA C array is zero. Second, some temporary array variables are replaced by scalar variables re-computed on GPU memory as needed and stored in local register memory; we call these scalarable. This is done because re-calculating values in GPU threads (i.e., local register memory) is faster than transferring them from a relatively slower global memory.
The third area for special care relates to the handling of the horizontal grid points $(i, j)$ inside the kernel. When translating $\mathrm{C}$ programs to CUDA $\mathrm{C}$ programs, the loops for spatial grid points $(i, j)$ are replaced by index computations using thread and block indices:

$i=$ threadIdx.x + blockId $x . x \times$ blockDim.x

$j=$ blockIdx.y,

where threadIdx and blockIdx are thread index and block index, respectively, and blockDim the dimensional size of a block. Each grid point $(i, j)$ represents a thread in CUDA $\mathrm{C}$ programs. Hence, there are two purposes for decomposing the domain in this way. One is to make the execution in each thread independent from one another, and the other is to compute values for all vertical levels (i.e., all $k$ components in the CUDA C programs) in one spatial grid position, $(i, j)$. There are no interactions among horizontal grid points, according to the physical model described in Sect. 2, which means that this computationally efficient thread and block layout is permissible.

Fourth, one way to check whether a kernel is launched successfully is to call cudaGetLastError(). If the kernel launch fails, this command would report error messages right after the CUDA kernel launch. Finally, once a kernel is successfully launched, to obtain the correct GPU execution runtime, command cudaThreadSynchronize() must be called.

The default compiler options from WRF were used to compile the Fortran and $\mathrm{C}$ versions of the YSU PBL scheme. For Fortran programs, we used gfortran along with compiler options: -O3 -ftree-vectorize -ftree-loop-linear -funrollloops. For $\mathrm{C}$ programs, we used gcc with compiler options: -O3 -ftree -vectorize -ftree-loop-linear -funroll-loops -lm. First, however, we verified that the outputs of $\mathrm{C}$ programs were identical to those of the Fortran programs using the same level of compiler optimization.

When the first CUDA C version of the YSU PBL scheme, directly translated from $\mathrm{C}$ version without any optimization, was ready, we examined features of the original Fortran programs to discover further optimization opportunities. We will present the evolution of this scheme from a CPU basis to a parallel GPU basis in the next section.

\section{Development of GPU-based YSU PBL scheme}

\subsection{Premise for optimizing GPU-based YSU PBL scheme}

To perform the GPU-based YSU PBL scheme, the CUDA C programs were compiled using nvcc (NVIDIA CUDA compiler) version 6.0 and executed on one Tesla K40 GPU with compute capability 3.5 . The compiler options are $-\mathrm{O} 3-g p u$ architecture sm 35 -fmad=false - m64 -maxrregcount 63 restrict. The value of 63 means the number of registers per thread, which was randomly picked at present. Besides, the 
Table 1. GPU runtime and speedup as compared to one singlethreaded CPU core for the first CUDA C version of the YSU PBL scheme, where block size $=64$ and registers per thread $=63$ are used.

\begin{tabular}{lccc}
\hline & CPU runtime & GPU runtime & Speedup \\
\hline 1 CPU core & $1800.0 \mathrm{~ms}$ & & \\
Non-coalesced & & $36.0 \mathrm{~ms}$ & $50.0 \times$ \\
Coalesced & & $34.2 \mathrm{~ms}$ & $52.6 \times$ \\
\hline
\end{tabular}

thread block size (i.e., threads per block) was chosen as 64 at this stage. The effects of block size and registers per thread for performing this scheme will be discussed in Sect. 4.5 and 4.6, respectively. Table 1 lists the runtime and speedup of this first-version GPU-based scheme, where the speedup is calculated as GPU runtime as compared to its CPU counterpart running on 1 CPU core of an Intel Xeon E5-2603. The same definition of speedup calculation is used in subsequent discussions.

Before we go further to present our optimizations on the GPU-based YSU PBL scheme, a couple of things are worth mentioning. The computation of this scheme is merely one intermediate module of the entire WRF module. When WRF is fully implemented on GPUs, the inputs of this intermediate module will not have to be transferred from CPU memory. Instead, they will be the results from previous WRF module. Similarly, outputs of this will be inputs to the next WRF module. Hence, transfers to/from CPU memory are not involved in this intermediate module during its computation. In the studies presented in Sect. 4.1-4.8, I/O transfer timings are turned off. The results of computing performance with I/O transfer and multi-GPUs will be given in Sect. 4.9. In each evolution of optimization, the correctness of CUDA C outputs must be verified in comparison to the original Fortran outputs.

\subsection{Optimization with more $\mathrm{L} 1$ cache}

For each Tesla K40 GPU (see Fig. 2), every SMX has a data cache, which is shared among CUDA cores. The data cache can be configured as $16 \mathrm{~KB}$ software-managed cache called shared memory and $48 \mathrm{~KB}$ hardware cache (L1) or the other way around, or both can share the memory equally, i.e. $32 \mathrm{~KB}$ each.

To employ more L1 cache than shared memory, a command cudaFuncCachePreferL1 was launched in our CUDA $\mathrm{C}$ programs. In contrast, less $\mathrm{L} 1$ cache than shared memory is indicated without this command. Figure 5 depicts the memory allocation between L1 cache and shared memory with and without launching the L1 cache command.

Starting with the first CUDA C version of the YSU PBL scheme, the computing performances with L1 cache command was found to be better than that without this command, while the latter performance was noticed to be almost the

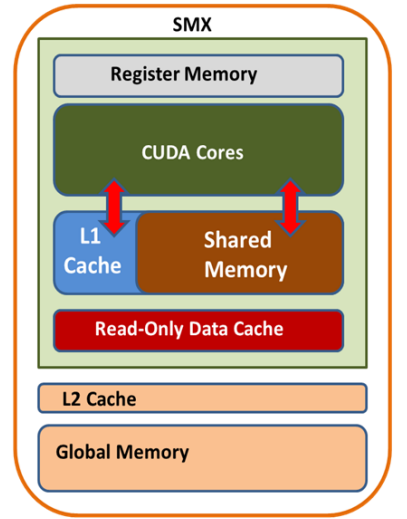

Command cudaFuncCachePreferL1 Is Not launched

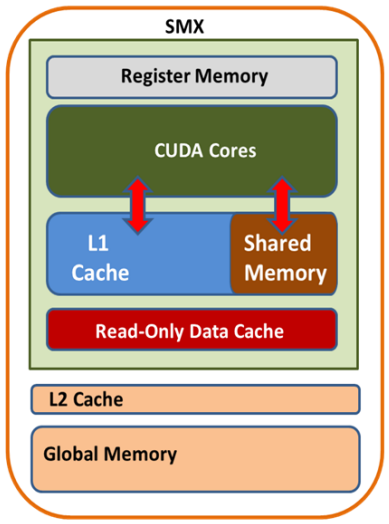

Command cudaFuncCachePreferL1 Is launched
Figure 5. Memory allocation of L1 cache and shared memory by launching cudaFuncCachePreferL1 or not.

Table 2. GPU runtime and speedup as compared to one singlethreaded CPU core for the first CUDA $\mathrm{C}$ version of the YSU PBL scheme after the L1 cache command cudaFuncCachePreferL1 is applied, where block size $=64$ and registers per thread $=63$ are used.

\begin{tabular}{lccc}
\hline & CPU runtime & GPU runtime & Speedup \\
\hline 1 CPU core & $1800.0 \mathrm{~ms}$ & & \\
Non-coalesced & & $34.3 \mathrm{~ms}$ & $52.5 \times$ \\
Coalesced & & $33.0 \mathrm{~ms}$ & $54.5 \times$ \\
\hline
\end{tabular}

same as that using cudaFuncCachePreferShared command. This suggests that usage of more L1 cache helps to speed up the CUDA C programs for this scheme. The GPU runtime and speedup are summarized in Table 2 after L1 cache command cudaFuncCachePreferLl is launched.

\subsection{Optimization with scalarizing temporary arrays}

Due to the parallelism architecture in CUDA programs, each thread must have its own local copy of the temporary array data. For instance, for 3-D arrays, when they are converted from CPU-based implementation to GPU-based implementation, they are retained as 3-D, but one-dimensional (1-D) and 2-D arrays that involve with $k$ (height) components must be re-arranged as 3-D arrays in GPU-based implementation; otherwise, the access to the contents of the arrays could be some other computed values from other threads. One special case is that the 2-D arrays with $(i, j)$ elements, are still kept as arrays with $(i, j)$ elements because what each thread deals with is the grid point $(i, j)$. By arranging arrays in this way, which is necessary in GPU-based implementation, memory usage is considerably increased, and the computing performance degraded. Figure 6 illustrates the concepts of such array re-arrangement. 


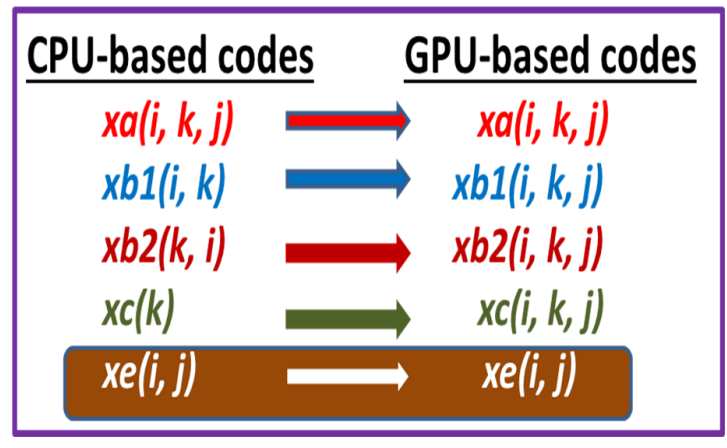

Figure 6. Illustration for re-arranging arrays in CPU-based implementation to 3-D and 2-D arrays in GPU-based implementation, where regular mathematical array syntax is used to express the arrays.

Table 3. GPU runtime and speedup as compared to 1 singlethreaded CPU core after further improvement with replacing temporary arrays by scalar variables, where block size $=64$ and registers per thread $=63$ are used.

\begin{tabular}{lccc}
\hline & CPU runtime & GPU runtime & Speedup \\
\hline 1 CPU core & $1800.0 \mathrm{~ms}$ & & \\
Non-coalesced & & $22.3 \mathrm{~ms}$ & $80.7 \times$ \\
Coalesced & & $21.4 \mathrm{~ms}$ & $84.1 \times$ \\
\hline
\end{tabular}

One solution to such a problem is to reduce the use of loop operation in the CUDA programs by merging several loop operations into one single loop operation. With this approach, some scalarable temporary arrays are replaced by scalar variables. Owing to the structure of the WRF model, this approach can only be applicable to those scalarable temporary arrays in the vertical-level (i.e., $k$ ) components, not in the grid-point component (i.e., $i$ or $j$ ). The scalar variables are re-computed in fast local memory, rather than in the slower global memory, in order to reduce the access time. This approach of diminishing the number of loop operations is applicable to CPU-based programs. However, the computation are still executed in 1 single-threaded core by looping through all horizontal grid points $(i, j)$, and it would not speed up the computing performance much. In contrast, this loop-reduction approach is extremely useful for GPU-based programs due to its multi-threaded nature.

Figure 7 illustrates the concept presented here. This reduces the transferring of data from global memory and delivers significant time savings. For this YSU PBL scheme, such replacement of temporary arrays by scalar variables drops the temporary arrays from 68 down to only 14 arrays, and apparently enhances the computing performance by a lot. Table 3 summarizes the GPU runtime and speedup after scalarizing most of temporary arrays.
Table 4. GPU runtime and speedup as compared to 1 singlethreaded CPU core after releasing height dependence, where block size $=64$ and registers per thread $=63$ are used.

\begin{tabular}{lccc}
\hline & CPU runtime & GPU runtime & Speedup \\
\hline 1 CPU core & $1800.0 \mathrm{~ms}$ & & \\
Non-coalesced & & $10.74 \mathrm{~ms}$ & $167.6 \times$ \\
Coalesced & & $9.29 \mathrm{~ms}$ & $193.8 \times$ \\
\hline
\end{tabular}

Table 5. GPU runtime and speedup as compared to 1 singlethreaded CPU core for data transfer between CPU and GPUs, where block size $=64$ and registers $/$ thread $=63$ are used.

\begin{tabular}{lcccc}
\hline CPU runtime & $1800.0 \mathrm{~ms}$ & & & \\
\hline GPU runtime & Non-coalesced & Speedup & Coalesced & Speedup \\
\hline Host to device & $27.26 \mathrm{~ms}$ & & $30.69 \mathrm{~ms}$ & \\
Kernel execution & $10.74 \mathrm{~ms}$ & $167.6 \times$ & $9.29 \mathrm{~ms}$ & $193.8 \times$ \\
Device to host & $10.83 \mathrm{~ms}$ & & $20.39 \mathrm{~ms}$ & \\
Kernel + Sync I/O & $48.83 \mathrm{~ms}$ & $36.9 \times$ & $60.37 \mathrm{~ms}$ & $29.8 \times$ \\
With Async I/O & $52.55 \mathrm{~ms}$ & $34.3 \times$ & $54.66 \mathrm{~ms}$ & $32.9 \times$ \\
\hline
\end{tabular}

\subsection{Optimization with height dependence release}

The remaining 14 local arrays present difficulties because the $k$ components of the vertical heights are not independent with one another. This can be seen from Eq. (22) when one tries to solve the diffusion equations for those prognostic variables $\left(C, u, v, \theta, q_{v}, q_{c}, q_{i}\right)$, where there is dependence in the vertical $z$ level (i.e., $k$ component in the codes). These 14 local arrays are involved in the final solution calculation for those prognostic variables. For the first part of these 14 array variables involved, the calculation of the $k$ th component depends on the input of the $(k-1)$ th component. This is to calculate the contents inside the brackets in Eq. (22), which is a differential equation as a function of the vertical height and is related to calculations in Eqs. (1)-(21). For the second part, the calculation of the $k$ th component needs inputs from the $(k+1)$ th component. This is to carry out the final results for those prognostic variables, which is again a derivative equation with respect to the vertical height.

About one-third of the original Fortran programs appears to involve dependencies among $(k-1)$ th, $k$ th, and $(k+1)$ th components. Figure 8 describes the conceptual idea for how to release the height dependence in order to reduce the access time to the global memory. This is the most time-consuming part in the physical programs. Table 4 gives the GPU runtime and speedup after the release of the height dependence.

\subsection{Impact of block size on GPU-based YSU PBL scheme}

The impact of the thread block size on the computing performance of the GPU-based YSU PBL scheme was evaluated by varying the block size while keeping the registers per thread 


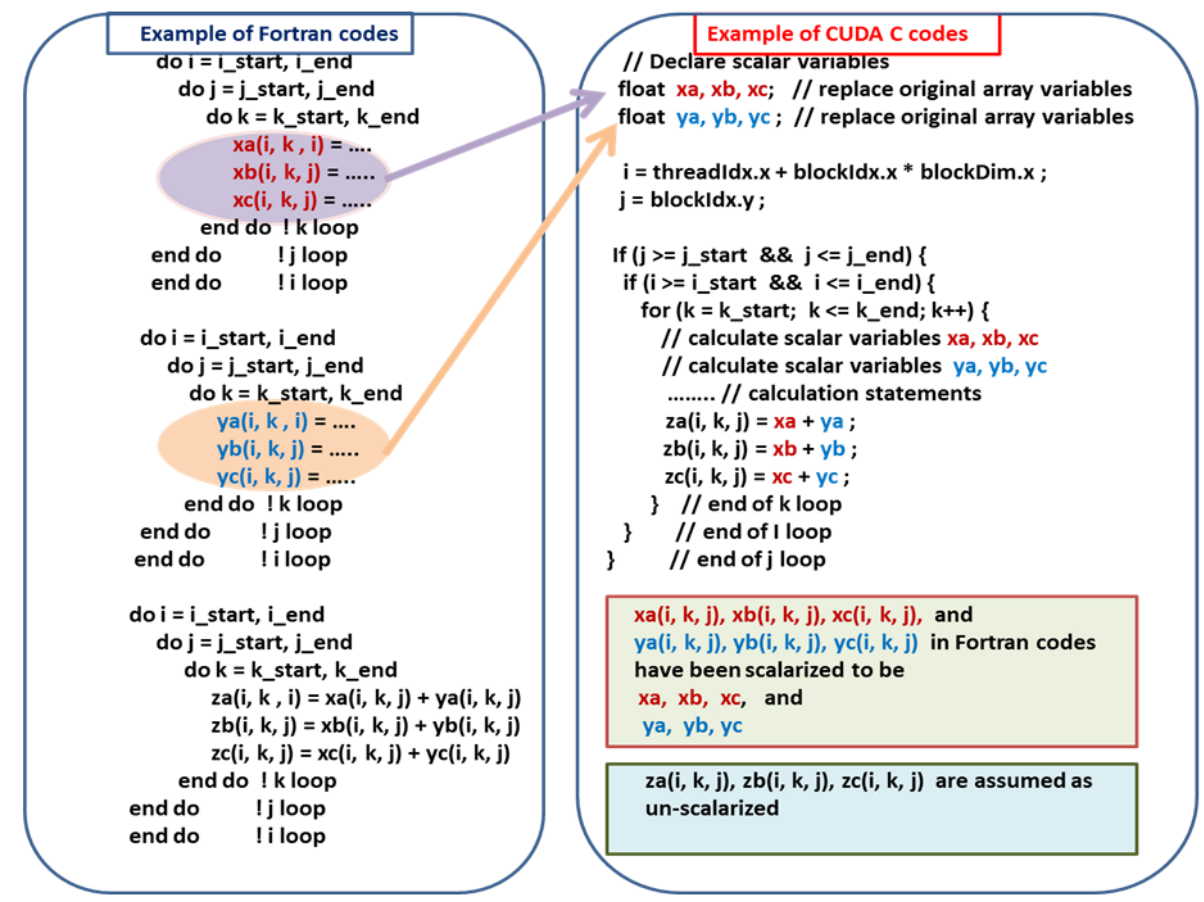

Figure 7. Illustration of scalarizing temporary arrays by scalar variable in order to re-calculate values in the local memory rather than transferring them from global memory to local memory. In this illustration, $x a, x b, x c, y a, y b, y c$ are assumed to be scalarable, but $z a, z b, z c$ are assumed to be un-scalarizable. Note that the regular mathematical array syntax is used to express the arrays.

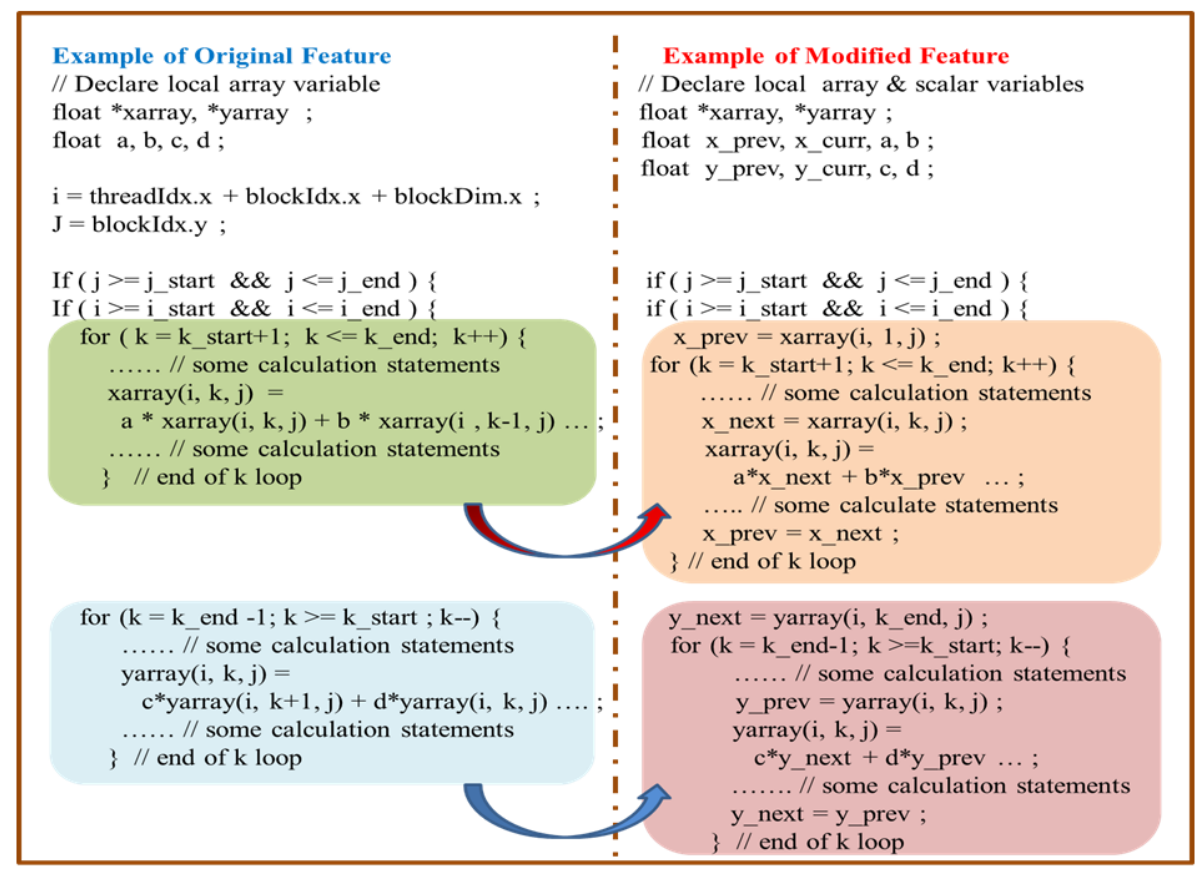

Figure 8. Illustration for how to release height dependence in order to reduce the access time to global memory for those temporary arrays which have dependence among the $(k-1)$ th, $k$ th, and $(k+1)$ th components. The left (right) figure is the original (modified) CUDA program.

at 63. Figure 9 shows the speedup of the optimized CUDA C programs (see Sect. 4.2-4.4) vs. the block size for cases with and without coalesced memory access. To obtain this plot, 16 executions of the CUDA $\mathrm{C}$ programs are executed in a row for each given block size. Excluding the first three runtimes due to unstable computing performance, the remaining run- 
Table 6. (a) Comparison of maximal RMSE for those variables having different outputs from Fortran outputs for four different combination cases, where "Spec" stands for special function. (b) Comparisons of speedups using one GPU for four different combination cases, where "Spec" stands for special functions. Note that the optimized GPU-based YSU PBL scheme along with block size $=64$ and register/thread $=63$ is used in these comparison.

\begin{tabular}{lcccc}
\hline (a) Variables & A & B & C & D \\
\hline Horizontal velocity $u$ component & $2.5 \times 10^{-04}$ & $1.1 \times 10^{-08}$ & $4.1 \times 10^{-06}$ & 0 \\
Horizontal velocity $v$ component & $2.3 \times 10^{-04}$ & $1.0 \times 10^{-08}$ & $1.8 \times 10^{-06}$ & 0 \\
Potential temperature $(\theta)$ & $5.2 \times 10^{-05}$ & $1.0 \times 10^{-07}$ & $1.1 \times 10^{-06}$ & 0 \\
Mixing ratio of water vapor $\left(q_{\mathrm{v}}\right)$ & $4.3 \times 10^{-08}$ & $1.3 \times 10^{-11}$ & $1.5 \times 10^{-09}$ & 0 \\
Mixing ratio of cloud water $\left(q_{\mathrm{c}}\right)$ & $1.3 \times 10^{-08}$ & $4.7 \times 10^{-14}$ & $1.9 \times 10^{-12}$ & 0 \\
Exchange coefficient & $3.0 \times 10^{-00}$ & $2.9 \times 10^{-06}$ & $1.8 \times 10^{-03}$ & 0 \\
PBL height & $3.9 \times 10^{+02}$ & $1.3 \times 10^{-04}$ & $2.8 \times 10^{-02}$ & 0 \\
\hline (b) GPU-Based code & & & & \\
running conditions & $\mathrm{A}$ & $\mathrm{B}$ & $\mathrm{C}$ & $\mathrm{D}$ \\
\hline Non-coalesced & $208.9 \times$ & $151.6 \times$ & $193.1 \times$ & $167.6 \times$ \\
Coalesced & $311.2 \times$ & $167.7 \times$ & $250.3 \times$ & $193.8 \times$ \\
\hline
\end{tabular}

A: - use_fast_math + CUDA C built-in Spec; B: -fmad=false + CUDA C built-in Spec; C: -use_fast_math + our own Spec; D: - fmad=false + our own Spec.

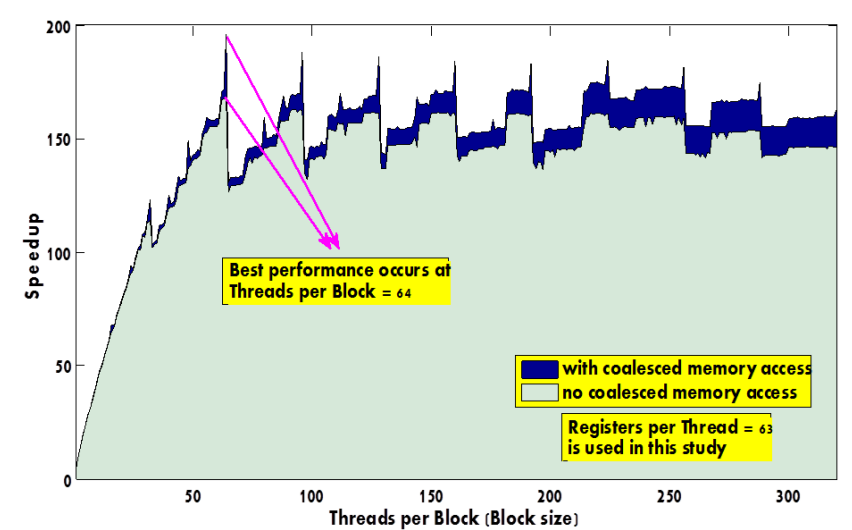

Figure 9. Speedup vs. threads per block (block size) for optimized GPU-based YSU PBL scheme.

times are averaged and used for the average speedup calculation for the given block size. From this study, it was found that the block size of 64 could produce the best performance; this is what we used in Sect. 4.1-4.4.

In addition, when the block size is a multiple of 32 threads, i.e., a warp, it was found that the computing performance is better than the neighboring block sizes, which forms a cycle of oscillation every 32 threads. This is understandable because threads of 32 are grouped together and the multiprocessor issues the same instruction to all the threads in a warp in order to make execution more efficient. This is one of the merits of the GPU architecture.

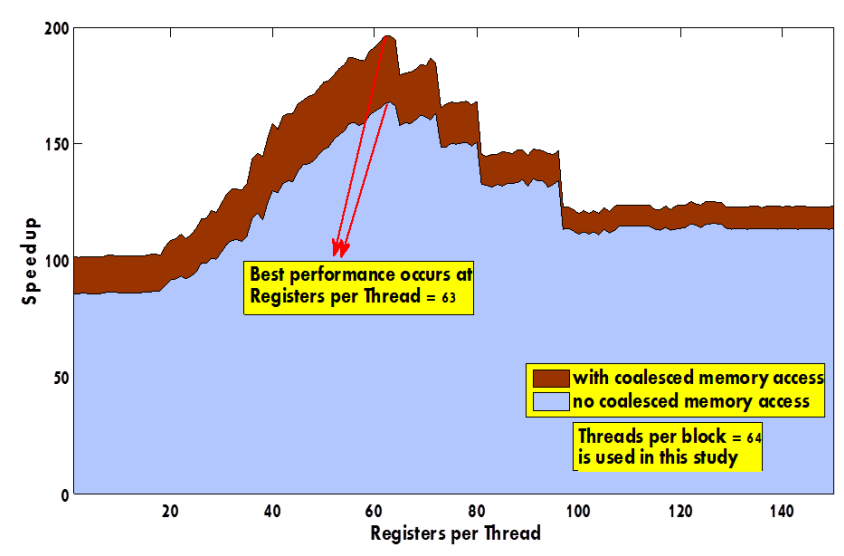

Figure 10. Speedup vs. registers per thread for optimized GPUbased YSU PBL scheme.

\subsection{Impact of registers per thread on GPU-based YSU PBL scheme}

By keeping the block size at 64, the optimized CUDA C version of the YSU PBL scheme was studied for speedup vs. the number of registers per thread. Similar to the approach presented in the previous section, given a number of registers per thread, 16 executions of the CUDA C programs are performed and only the last 13 runtimes are used in calculating the average speedup. The results are displayed in Fig. 10 for cases with and without coalesced memory access. This figure shows that the optimal computing performance occurs at 63 registers per thread for this scheme, and the speedups seem to keep dropping beyond this number of registers. 
Table 7. Results of runtime and speedup for CPU-based and optimized GPU-based YSU PBL scheme, where block size $=64$ and registers per thread $=63$, along with compiler option - fmad $=$ false, as well as our own modified special functions, are used.

\begin{tabular}{lcccc}
\hline & \multicolumn{1}{c}{ CPU runtime } & \multicolumn{2}{c}{ Speedup } \\
\hline 1 CPU core & \multicolumn{2}{c}{$1800.0 \mathrm{~ms}$} \\
One CPU socket & \multicolumn{2}{c}{$509.0 \mathrm{~ms}$} & \multicolumn{2}{c}{$3.5 \times$} \\
\hline \multicolumn{4}{c}{ GPU runtime } & \multicolumn{2}{c}{ Speedup } \\
\hline Without I/O & Non-coalesced & Coalesced & Non-coalesced & Coalesced \\
\hline 1 GPU & $10.74 \mathrm{~ms}$ & $9.29 \mathrm{~ms}$ & $167.6 \times$ & $193.8 \times$ \\
2 GPUs & $5.80 \mathrm{~ms}$ & $4.99 \mathrm{~ms}$ & $310.9 \times$ & $360.6 \times$ \\
\hline \multicolumn{2}{c}{ GPU runtime } & \multicolumn{2}{c}{ Speedup } \\
\hline Async I/O & Non-coalesced & Coalesced & Non-coalesced & Coalesced \\
\hline 1 GPU & $52.55 \mathrm{~ms}$ & $54.66 \mathrm{~ms}$ & $34.3 \times$ & $32.9 \times$ \\
2 GPUs & $35.88 \mathrm{~ms}$ & $46.31 \mathrm{~ms}$ & $50.2 \times$ & $38.9 \times$ \\
\hline
\end{tabular}

\subsection{Data transfer between CPU and GPUs}

Usually the time consumed by kernel execution is less than the time occupied by the data transfer between CPU (i.e., host) and GPUs (i.e., devices). Using the optimized CUDA $\mathrm{C}$ programs of the YSU PBL scheme along with the optimal registers/thread $=63$ and block size $=64$ just obtained previously, Table 5 lists the GPU runtimes for cases with and without coalesced memory access. In the YSU PBL scheme, there are 17 3-D array variables, 17 2-D array variables, and two 1-D array variables needed to be input from CPU to GPUs, amounting to 326475352 bytes. For the outputs, this scheme needs a transfer of seven 3-D array variables and seven 2-D array variables from GPUs back to the CPU, corresponding to 134430912 bytes in total. The data size of the outputs is about $41 \%$ of that of the inputs, which indicates a rough consistency with the runtime taken by device-to-host data transfer as compared to that by host-to-device data copy. This is shown in Table 5, where the I/O transfer involved here is synchronous memory access.

This supports the general finding that data transfer between CPU and GPUs takes up a lot of time, and apparently is a limiting factor for the speedup. Since the computation of this scheme is only one intermediate module of the entire WRF model, what we are more interested in is its speedup with no I/O transfer, for the reasons given in Sect. 4.1.

However, when I/O transfer is considered, the use of asynchronous memory access to overlap CUDA kernel execution with data transfer can be applied. Each Tesla K40 has one copy engine and one kernel engine, allowing data transfer and kernel execution to overlap. As commands pipeline, streams execute commands in a manner of first-in-first-out (FIFO) order. The stream arrangement would result in overlapping CPU-to-GPU and GPU-to-CPU memory transfers and kernel execution on GPUs with two copy engines. A diagram that depicts the execution timeline of the YSU PBL computation process is illustrated in Fig. 11, where the illustrated three streams are in different colors. For comparison, when asynchronous memory access is taken into account, the results of computing performance are listed in the last row of Table 5.

\subsection{Output comparison between CPU-based and GPU-based programs}

It was found that the GPU-based programs of the YSU PBL scheme were sensitive to precision. Two key issues we encountered were special functions such as $\operatorname{powf}(), \exp f()$, sqrtf(), and the compiler option for the math calculation. First, when $\mathrm{C}$ programs were converted to CUDA C programs, the special functions taken from GNU C library source codes must be modified to be used by GPU devices; otherwise, incorrect outputs will emerge (in comparison to Fortran outputs), if CUDA C built-in special functions were used. Second, when the compiler option of math calculation, -use_fast_math, was employed to compile CUDA C programs, incorrect outputs of some variables would appear, again, as compared to Fortran outputs. This compiler option made the CUDA C programs run faster, but it could not produce outputs identical to the Fortran outputs. Thus, another math-calculation option, -fmad=false, was chosen.

A mathematical function causing the highest unit in the last place $(u l p)$ difference between GNU C math library and CUDA $\mathrm{C}$ functions is the power function. In Fig. 12, ulp differences for the power function in GPU CUDA C library with and without fast math option are compared to the GNU C mathematical library. The library function for the power function is $\operatorname{powf}(x, y)$. In the plot, $x$ starts from the value 500.37420654296875 and $y$ has a constant value of 0.9505939483642578125 . The spacing of the value $x$ in the plot is non-equal as each consecutive value is derived from the previous value by adding one $\boldsymbol{u l p}$ to the previous value. 


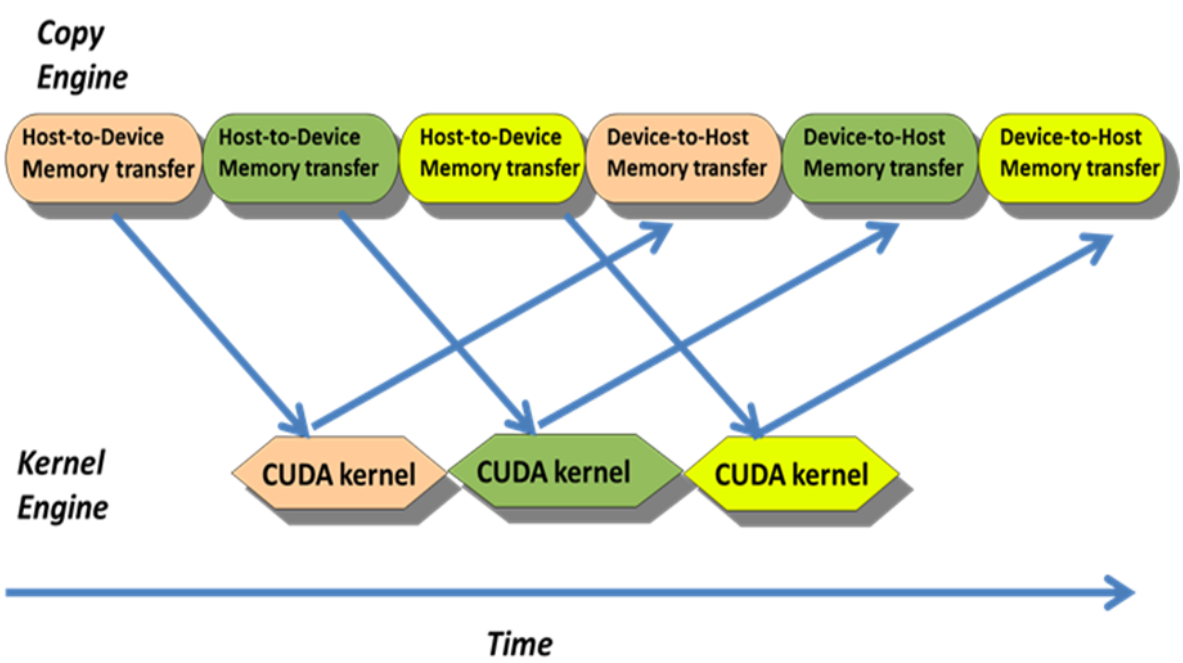

Figure 11. Asynchronous memory transfer among host-to-device memory transfer, GPU kernel execution, and device-to-host memory transfer.

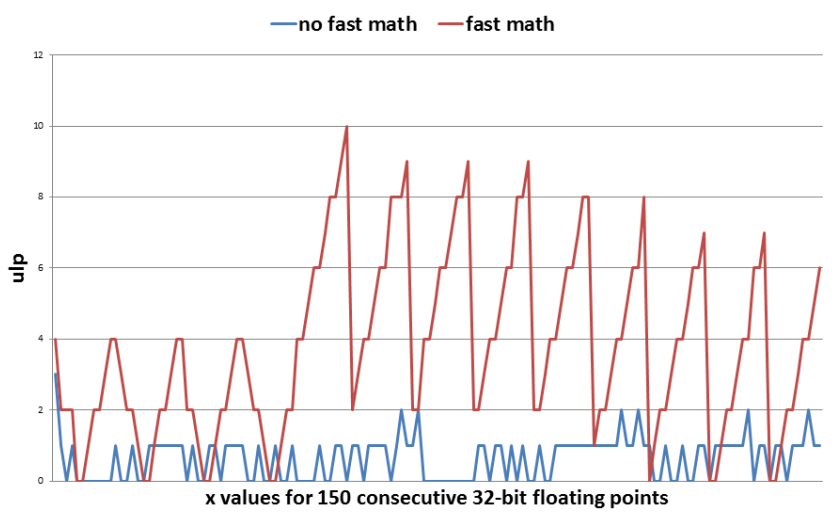

Figure 12. Unit in the last place error for power function. Both fast math and no fast math GPU options are compared to the GNU C math library.

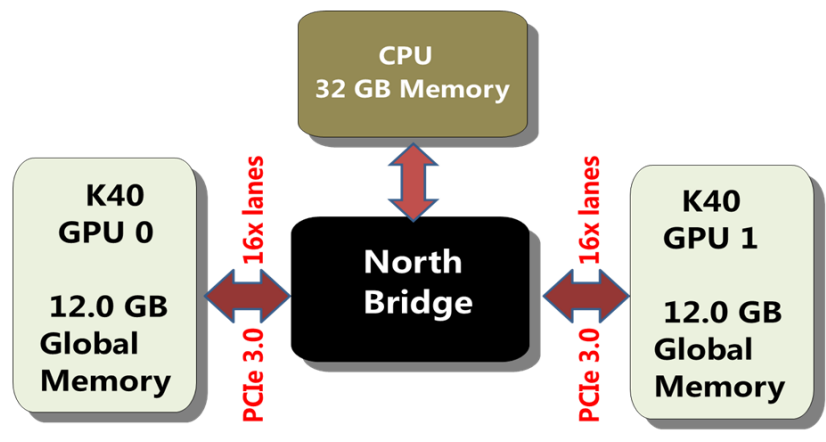

Figure 13. Schematic diagram of our multi-GPU setup.

Thus, the plot shows $u l p$ values for 150 consecutive 32-bit floating point values starting from the first value. For these example values, the $u l p$ ranges from 0 to 10 with fast math.
Without fast math, the maximum ulp value is 3. Due to error cascading effects in the chaotic YSU PBL algorithm, an ulp error of 3 is capable of causing a large error in the final output. In order to get exactly the same results for the math functions on GPU and CPU, we adopted GNU C math libraries for GPU execution by adding CUDA C device execution qualifiers to the existing $\mathrm{C}$ source code.

The root-mean square error (RMSE) is employed to make a comparison between the CUDA C outputs of a variable and Fortran outputs of the same variable by aggregating over all grid-point elements of this variable. The comparison of the maximal RMSE for those variables having different CUDA C outputs from Fortran outputs are listed in Table 6a. In addition, as mentioned above, the fast math compiler option, -use_fast_math, may allow us to execute much faster, but it produces less accurate results. Furthermore, the built-in GPU CUDA C special functions are also able to make programs run faster, but again with less accuracy. Table $6 \mathrm{~b}$ lists the comparison of the speedups for four different running situations. From these studies, it is found that the option fmad=false along with our own modified special functions can make our CUDA C programs produce outputs identical to Fortran outputs, albeit with less speedup.

\subsection{Multi-GPU implementation}

Our multi-GPU setup is displayed in Fig. 13. In this setup, there are two Tesla K40 GPU cards, each of which has one GPU. Multi-GPU implementation of the YSU PBL scheme is performed by computing several contiguous $j$-dimensional values in the arrays within the same GPU. With asynchronous access taken into account, the optimal numbers of $j$ dimensions for transfers between CPU and GPUs were found to be 26 for use of one or two GPUs. 
Using the optimal block size $=64$ and registers per thread $=63$, the YSU PBL scheme was executed on our multi-GPUs setup. Table 7 lists the computation times and speedups for single GPU and two GPUs with/without I/O transfer and with/without coalesced memory access. We also ran the Fortran programs using one CPU socket (4 cores), and the runtime and speedup are also listed in the same table.

\section{Summary and future work}

In this paper, we develop an efficient parallel GPU-based YSU PBL scheme of the WRF model using NVIDIA Tesla K40 GPUs. From our study, the communication bandwidth of data transfer is one of the main limiting factors for computing performance of CUDA codes on GPUs. This limitation holds true for other WRF schemes as well. To ameliorate this problem, several crucial code changes were made to improve the computing performance. For example, we launched a L1 cache with more memory than shared memory, some temporary arrays have been scalarized, and the dependence among the vertical-level components has been freed. In addition, the effects of threads per block and registers per thread on the GPU-based YSU PBL scheme were studied. We also discussed how the compiler options for math calculation would affect the outputs of the GPU-based programs. At the end, we came up with an optimized GPU-based YSU PBL scheme with outputs identical to the CPU-based Fortran programs.

When WRF is fully implemented on GPUs, the implementation of input/output transfers between CPU and GPU(s) will not be needed for each intermediate module. Instead, only inputs to the first WRF module have to be transferred from CPU to GPU(s), and only the outputs from the last WRF module will be transferred from GPU(s) to CPU. The YSU PBL scheme is only one intermediate module of the entire WRF model. Therefore, the speedups for the YSU PBL scheme are expected to be close to the results presented in the cases without I/O transfer rather than to those with I/O transfer.

Using one NVIDIA Tesla K40 GPU in the case without I/O transfer, our optimization efforts on the GPU-based YSU PBL scheme can achieve a speedup of $193 \times$ with respect to 1 CPU core, whereas the speedup for one CPU socket (4 cores) with respect to $1 \mathrm{CPU}$ core is only $3.5 \times$. We also ran the CPU-based code on $1 \mathrm{CPU}$ core using exactly the same optimization along with height dependence release as the GPUbased code, and its speedup is merely $1.5 \times$ as compared to its original Fortran counterpart. In addition, we can even boost the GPU-based speedup to $360 \times$ with respect to $1 \mathrm{CPU}$ core when two K40 GPUs are applied; in this case, 1 min of model execution on dual Tesla K40 GPUs will achieve the same outcome as $6 \mathrm{~h}$ of execution on a single core CPU.

Our future work is to continue accelerating other parts of WRF model using GPUs. Eventually, we expect to have a WRF model completely running on GPUs. This will provide a superior performance for weather research and forecasting in the near future.

Acknowledgements. This work is supported by the National Oceanic and Atmospheric Administration (NOAA) under grant no. NA10NES4400013. B. Huang would like to thank Stanley Posey of NVIDIA for donating Tesla K40 GPUs. The authors are also grateful to James E. Davies of Space Science and Engineering Center at University of Wisconsin-Madison for professional editing of this manuscript. In addition, we are indebted to the anonymous referees for providing insightful comments and constructive suggestions to improve the quality of this paper.

Edited by: A. Sandu

\section{References}

Betts, A., Hong, S.-Y., and Pan, H.-L.: Comparison of NCEPNCAR reanalysis with 1987 FIFE data, Mon. Weather Rev., 124, 14801498, 1996.

Bright, D. R. and Mullen, S. L.: The sensitivity of the numerical simulation of the southwest monsoon boundary layer to the choice of PBL turbulence parameterization in MM5, Weather Forecast., 17, 99-114, 2002.

Continental US (CONUS): WRF V3 Parallel Benchmark Page, Single domain, medium size, $12 \mathrm{~km}$ CONUS, Oct. 2001, available at: http://www2.mmm.ucar.edu/wrf/WG2/benchv3/\#_ Toc212961288, last access: 18 June 2008.

Durran, D. R. and Klemp, J. B.: The effects of moisture on trapped mountain lee waves, J. Atmos. Sci., 39, 2490-2506, 1982.

Hong, S.-Y. and Pan, H.-L.: Nonlocal boundary layer vertical diffusion in a Medium-Range Forecast model, Mon. Weather Rev., 124, 2322-2339, 1996.

Hong, S.-Y., Noh, Y., and Dudhia, J.: A new vertical diffusion package with an explicit treatment of entrainment processes, Mon. Weather Rev., 134, 2318-2341, 2006.

Horn, S.: ASAMgpu V1.0 - a moist fully compressible atmospheric model using graphics processing units (GPUs), Geosci. Model Dev., 5, 345-353, doi:10.5194/gmd-5-345-2012, 2012.

Kim, J. and Mahrt, L.: Simple formulation of turbulent mixing in the stable free atmosphere and nocturnal boundary layer, Tellus A, 44, 381-394, 1992.

Klingbeil, G., Erban, R., Giles, M., and Maini, P. K.: Fat versus thin threading approach on GPUs: application to stochastic simulation of chemical reactions, IEEE T. Parall. Distr., 23, 280-287, doi:10.1109/TPDS.2011.157, 2012.

Louis, J. F.: A parametric model of vertical eddy fluxes in the atmosphere, Bound.-Lay. Meteorol., 17, 187-202, 1979.

Mielikainen, J., Huang, B., Huang, H.-L. A., Goldberg, M. D., and Mehta, A.: Speeding up the computation of WRF double moment 6-class microphysics scheme with GPU, J. Atmos. Ocean. Tech., 30, 2896-2906, doi:10.1175/JTECH-D-12-00218.1, 2013.

Moeng, C. H. and Sullivan, P. P.: A comparison of shear and buoyancy-driven planetary boundary layer flows, J. Atmos. Sci., 51, 999-1022, 1994. 
Noh, Y., Cheon, W. G., Hong, S.-Y., and Raasch, S.: Improvement of the K-profile model for the planetary boundary layer based on large eddy simulation data, Bound.-Lay. Meteorol., 107, 401427, 2003.

NVIDIA: Tesla GPU Accelerators, available at: http://www.nvidia.com/content/tesla/pdf/ NVIDIA-Tesla-Kepler-Family-Datasheet.pdf, last access: 13 October 2013a.

NVIDIA: Tesla K40 vs. K20X GPU, available at: http: //blog.xcelerit.com/benchmarks-nvidia-tesla-k40-vs-k20x-gpu/, last access: 20 November 2013b.

Overview of WRF Physics: available at: http://www2.mmm.ucar. edu/wrf/users/tutorial/201301/dudhia_physics.pdf, last access: 5 December 2014.

Paz, A. and Plaza, A.: Clusters versus GPUs for parallel automatic target detection in remotely sensed hyperspectral images, EURASIP J. Adv. Sig. Pr., 35, 18 pp., doi:10.1155/2010/915639, 2010.

Price, E., Mielikainen, J., Huang, M., Huang, B., Huang, H.-L. A., and Lee, T.: GPU-Accelerated Longwave Radiation Scheme of the Rapid Radiative Transfer Model for General Circulation Models (RRTMG), IEEE J. Sel. Top. Appl., 7, 3660-3667, doi:10.1109/JSTARS.2014.2315771, 2014.
Rustico, E., Bilotta, G., Herault, A., Negro, C. D., and Gallo, G.: Advances in Multi-GPU Smoothed Particle Hydrodynamics Simulations, IEEE T. Parall. Distr., 25, 43-52, doi:10.1109/TPDS.2012.340, 2014.

Siewertsen, E., Piwonski, J., and Slawig, T.: Porting marine ecosystem model spin-up using transport matrices to GPUs, Geosci. Model Dev., 6, 17-28, doi:10.5194/gmd-6-17-2013, 2013.

Song, C., Li, Y., and Huang, B.: A GPU-accelerated wavelet decompression system with SPIHT and Reed-Solomon decoding for satellite images, IEEE J. Sel. Topics Appl., 4, 683-690, doi:10.1109/JSTARS.2011.2159962, 2011.

Troen, I. and Mahrt, L.: A simple model of the atmospheric boundary layer sensitivity to surface evaporation, Bound.-Lay. Meteorol., 37, 129-148, 1986.

Wei, S.-C. and Huang, B.: GPU acceleration of predictive partitioned vector quantization for ultraspectral sounder data compression, IEEE J. Sel. Top. Appl., 4, 677-682, 2011.

Wu, X., Huang, B., Plaza, A., Li, Y., and Wu, C.: Real-time implementation of the pixel purity index algorithm for endmember identification on GPUs, IEEE Geosci. Remote S., 11, 955-959, 2014. 